\title{
Corrosion behaviour of reinforced concrete constructions in acid aggressive environments: case studies, laboratory tests and corrosion mechanisms
}

\section{Comportement à la corrosion des constructions en béton armé dans des milieux agressifs acides: études de cas, tests de laboratoire et mécanismes de corrosion}

\author{
Ioan Pepenar ${ }^{1}$ \\ ${ }^{1}$ ICECON GROUP, Centre de Recherche CERTINCON, Bucarest, Roumanie
}

\begin{abstract}
The paper presents the results of research on the corrosion behaviour of reinforced concrete elements which compose the bearing structure of some constructions subjected to the corrosive action of acid aggressive agents, after a long-term service in a strongly aggressive environment. To evaluate the damage state of reinforced concrete elements/constructions, both "in situ" investigation of constructions and laboratory tests on concrete samples extracted from some elements damaged by corrosion, using a specific investigation methodology were performed. The results of the research revealed the existence of a severe corrosion-induced damage of the structural elements, which affected the resistance, stability and durability of the constructions. Based on case studies on the service behaviour of reinforced concrete elements, there are pointed out the specific damages generated by the corrosive action of the acid aggressive agents, the causes of their occurrence and presents considerations on the mechanisms of the corrosion processes of concrete and steel reinforcement in acid aggressive environments. Finally, there were proposed intervention measures in order to assure normal service conditions of the reinforced concrete constructions located in such aggressive environments.
\end{abstract}

\begin{abstract}
Résumé. Le papier présente les résultats des recherches sur le comportement à la corrosion des éléments en béton armé qui constituent la structure de résistance des certaines constructions soumises à l'action corrosive des agents agressifs acides, après un service de longue durée dans le milieu très agressif. Afin d'évaluer l'état de dégradation des éléments/constructions en béton armé, suite à l'action corrosive des agents agressifs acides, on a utilisé une méthodologie spécifique d'investigation et de diagnostic, y comprenant des investigations "in situ" des constructions et des tests de laboratoire sur des échantillons de béton prélevés des certains éléments dégradés par corrosion. Les résultats des recherches ont révélé l'existence des dégradations graves par corrosion des éléments structuraux qui affectent la résistance mécanique, la stabilité et la durabilité des constructions. Sur la base des études de cas sur le comportement en service des éléments en béton armé, on met en évidence les dégradations spécifiques provoquées par l'action corrosive des agents agressifs acides, les causes de leur apparition et on présente des considérations sur les mécanismes des processus de corrosion du béton et des armatures en acier dans les milieux agressifs acides. Finalement, on présente des mesures d'intervention proposées pour assurer les conditions normales de service des constructions en béton armé situées dans tels milieux agressifs.
\end{abstract}

\section{Introduction}

La recherche du comportement des constructions en béton soumises à l'action des milieux agressifs, particulièrement des milieux acides et la réhabilitation des constructions dégradées par corrosion est d'actualité et d'une grande importance, tant du point de vue de la protection du milieu construit existant, de même que de la nécessité du perfectionnement continu de la conception, du calcul, de l'exécution et de la durabilité des 
constructions en béton armé et béton précontraint qui seront placées dans tels milieux agressifs.

$\mathrm{Au}$ cours des décennies récentes, ils ont rapporté de nombreux cas de durabilité insatisfaisante des constructions en béton situées dans des milieux agressifs, respectivement de dégradation des éléments structuraux sous l'action des agents agressifs après différents intervalles de service des constructions. L'expérience acquise après un grand nombre d'études et de recherches sur le comportement en service des constructions a montré que les plus fréquentes et les plus dangereux cas de dégradation par corrosion, avec des conséquences importantes sur leur résistance mécanique et stabilité se rencontrent dans les constructions de l'industrie chimique et d'autres industries où se déroulent des processus technologiques de nature chimique [1].

Par rapport à d'autres milieux agressifs, les milieux industriels contenant des agents agressifs à caractère acide sont parmi les milieux les plus agressifs et les plus complexes. Ces milieux présentent une agressivité spécifique et complexe sur les constructions en béton armé et béton précontraint, affectant tant le béton de même que l'armature en acier [2,3].

Dans le présent papier sont synthétisés les résultats des recherches sur le comportement à la corrosion acide des éléments en béton armé qui constituent la structure de résistance des certaines constructions après une service de longue durée dans des milieux très agressifs, les mécanismes des processus de corrosion du béton et des armatures en acier et les mesures d'intervention proposées pour assurer les conditions normales de service des constructions en béton armé situées dans tels milieux agressifs.

\section{2 Étude de cas}

On présente deux études de cas sur la dégradation par corrosion des constructions en béton armé situées dans milieux agressifs acides, l'un sur un bâtiment placé dans un milieu à base d'acide sulfurique et l'autre sur un bâtiment placé dans un milieu à base d'acide chlorhydrique.

Afin d'évaluer l'état de dégradation des éléments/constructions en béton armé, suite à l'action corrosive des agents agressifs acides, on a utilisé une méthodologie spécifique d'investigation et de diagnostic, y comprenant des investigations "in situ" des constructions et des tests de laboratoire sur des échantillons de béton prélevés des certains éléments dégradés par corrosion [4].

\subsection{Bâtiment de fabrication dans un milieu à base d'acide sulfurique}

Cette étude de cas se réfère à la dégradation par corrosion des éléments en béton armé d'un bâtiment industriel étagé, à l'intérieur duquel se déroule le processus technologique de fabrication du dioxyde de soufre liquide.

La structure de résistance du bâtiment est constituée de cadres transversales (poteaux et poutres) en béton armé monolithe, plancher intermédiaire technologique et éléments de toiture en béton armé. Indépendante de structure du bâtiment, il y a deux plateformes technologiques en béton armé de soutènement des équipements technologiques. Les plateformes sont réalisées sous forme des plaques qui s'appuient sur deux poutres longitudinales et des poteaux en béton armé avec propres fondations. On a effectué toute une série de réparations des dégradations subies lors le service du bâtiment, mais sans l'efficience attendue.

Les éléments en béton armé de l'intérieur du bâtiment de fabrication du dioxyde de soufre liquide ont été soumis à l'action de long durée d'un milieu fortement agressif constitué principalement de gaz et de vapeurs de dioxyde de soufre $\left(\mathrm{SO}_{2}\right)$, dont la concentration a été comprise entre 27 et $60 \mathrm{mg} / \mathrm{m}^{3}$ d'air, en présence de l'humidité élevée de l'air (supérieure à $75 \%$ pendant l'activité de production dans de l'intérieur du bâtiment), ainsi que des solutions concentrées d'acide sulfurique $\left(\mathrm{H}_{2} \mathrm{SO}_{4}\right)$ et d'acide sulfureux $\left(\mathrm{H}_{2} \mathrm{SO}_{3}\right)$. Suite aux dégradations du revêtement de sol en briques antiacides et des canalisations, ces solutions corrosives se sont infiltrées dans le sol, en affectant les fondations du bâtiment et en provoquant son tassement. Dans ces conditions, le milieu de l'intérieur du bâtiment présent une agressivité très fort sur les éléments en béton armé, conformément aux réglementations techniques en vigueur [5].

L'investigation de l'état technique du bâtiment de fabrication du dioxyde de soufre liquide, après plus de 30 ans de service dans le milieu très fortement agressif, a révélé l'existence des dégradations graves par corrosion de la structure de résistance entière, qui ont conduit à la réduction significative ou même à la perte de la capacité portante des certains éléments de construction et qui constituent un risque potentiel d'effondrement du bâtiment et d'accidents.

Les dégradations par corrosion des éléments de construction en béton armé consistent principalement en: - aux poteaux du bâtiment (figure 1):

- la dégradation en profondeur du béton par corrosion acide, notamment à la base des poteaux, en dépassant le niveau des armatures dans plusieurs cas et en laissant découvertes les armatures en contact direct avec le milieu agressif;

- la corrosion avancée des armatures longitudinales et transversales (étriers), avec des réductions significatives de section, jusqu'à leur disparition complète à la base des certains poteaux, par corrosion généralisée ("dissolution").

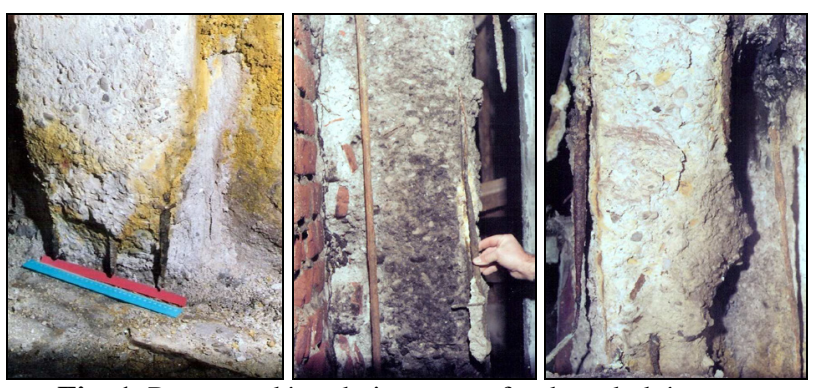

Fig. 1. Poteaux: dégradation en profondeur du béton par corrosion acide; réduction de la section de béton (plus de 50\%; 
corrosion avancée des armatures, leur disparition à la base des poteaux et au champ; armatures "flottantes".

Certains poteaux sont dans un état grave de dégradation, très dangereux, pour lesquels la réduction importante de la section de béton (plus de 50\%) est accompagnée de la disparition des armatures longitudinales et transversales (étriers) sur grandes zones/hauteurs, certaines armatures étant "flottantes". Il est évident que ces poteaux ne peuvent plus prendre les sollicitations sous des actions sismiques.

- aux poutres des cadres et des planchers:

- la dégradation en profondeur du béton par corrosion acide, en dépassant le niveau des armatures dans certains cas, pour les poutres des cadres et des planchers;

- la corrosion avancée des armatures longitudinales et transversales (étriers), avec des réductions significatives de section, jusqu'à la disparition des armatures, sur certaines zones;

- la dégradation complète des nervures du plancher de toiture dans les zones au-dessus des équipements technologiques;

- dégradations locales avancées de la plaque du plancher de toiture et du plancher technologique, surtout dans les zones à trous technologiques, à cause des infiltrations de solutions agressives; la dégradation en profondeur du béton sous l'action des gaz et vapeurs à caractère acide;

- aux plateformes technologiques (figure 2):

- dégradations graves des poteaux de soutènement des plateformes technologiques: la dégradation en profondeur du béton par corrosion acide, notamment à la base des poteaux, en dépassant le niveau des armatures dans certains cas; la corrosion avancée des armatures longitudinales et transversales (étriers), jusqu'à la disparition des armatures, sur certaines zones; il est évident que ces poteaux ne peuvent plus prendre les sollicitations sous des actions sismiques;

- dégradations graves des poutres et de la plaque des plateformes: la corrosion en profondeur du béton due aux écoulements des solutions acides, en dépassant le niveau des armatures dans plusieurs cas, jusqu'à la disparition des armatures, sur certaines zones.

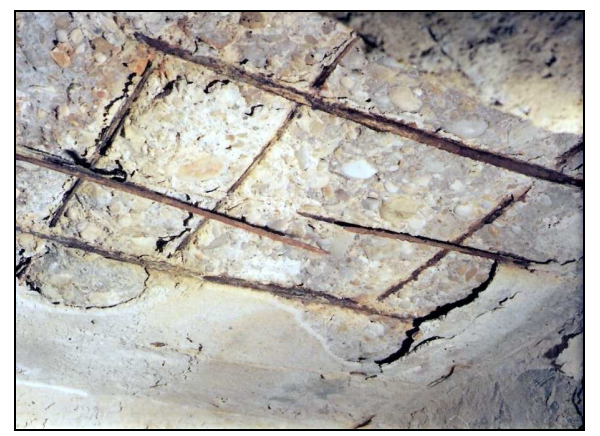

Fig. 2. Plateforme technologique: dégradation en profondeur du béton; corrosion avancée des armatures jusqu'à leur disparition, sur certaines zones.

La sécurité d'utilisation des plateformes technologiques est complètement compromise et il y a le risque de leur effondrement.

Les dégradations graves existantes sont dus au service de longue durée des éléments de construction dans un milieu fortement agressif, suite au fonctionnement défectueux des installations technologiques, y compris celles de capture et d'évacuation des agents agressifs (gaz, vapeurs et solutions à caractère fortement acide).

L'apparition et le développement des dégradations ont été aussi favorisé par l'absence des mesures efficientes de protection anticorrosive des éléments de construction par rapport à la nature et au degré d'agressivité du milieu de l'intérieur du bâtiment ainsi que de l'inefficience des travaux de maintenance du bâtiment et des installations technologiques.

Les processus de dégradation par corrosion des éléments de structuraux en béton armé sont dus à l'action spécifique des agents agressifs à caractère fortement acide, en affectant tant le béton (par corrosion acide) que les armatures en acier (par corrosion généralisée) et ayant comme résultat final la destruction complète des deux matériaux de construction.

Dans le cas du béton, sur l'effet destructive des ions d'hydrogène $\mathrm{H}^{+}$(corrosion acide) a été superposée l'effet agressif des ions $\mathrm{SO}_{4}{ }^{2-}$ (corrosion sulfatique), en provoquant des effets importantes de corrosion par l'expansion.

Étant donné l'état de dégradation très avancée du bâtiment, qui affectent la résistance mécanique, la stabilité et la sécurité d'utilisation du bâtiment, le niveau élevé d'infestation du sol avec des solutions fortement acides $\left(\mathrm{H}_{2} \mathrm{SO}_{4}\right)$, le degré avancé d'usure physique et morale de l'installation de fabrication du dioxyde de soufre liquide, on a proposé des mesures radicales d'intervention consistant en la démolition du bâtiment existant et la réalisation d'un nouveau bâtiment, conçu et construit conformément aux exigences fondamentales applicables aux ouvrages de construction [6] imposées par le processus technologique de fabrication, qui doit être modernisé également.

\subsection{Bâtiment de fabrication dans un milieu à base d'acide chlorhydrique}

Cette étude de cas se réfère à la dégradation par corrosion des éléments en béton armé d'un bâtiment industriel, à l'intérieur duquel se déroule le processus technologique de fabrication de l'acide chlorhydrique de synthèse par la combustion du chlore dans l'hydrogène sous vide.

La structure de résistance du bâtiment est constituée de deux cadres en béton armé monolithe, composés de deux poteaux avec section variable et d'une poutre de toiture, deux planchers technologiques et d'éléments de toiture en béton armé.

Les éléments en béton armé de l'intérieur du bâtiment de fabrication ont été soumis à l'action de long durée d'un milieu fortement agressif constitué principalement de gaz et de vapeurs de chlore $\left(\mathrm{Cl}_{2}\right)$ et d'acide chlorhydrique $(\mathrm{HCl})$, dont la concentration a variée entre 7,30 et 8,75 $\mathrm{mg} / \mathrm{m}^{3}$ d'air, en présence de l'humidité atmosphérique. En dehors des ces agents agressifs de l'intérieur du bâtiment, les éléments de construction ont été soumis à l'action d'agents agressifs dégagés de l'installation d'acide chlorhydrique située en plein air à proximité immédiate du bâtiment. Dans ces conditions, le milieu de l'intérieur 
du bâtiment présent une agressivité très fort sur les éléments de construction en béton armé, conformément aux réglementations techniques en vigueur [5].

L'investigation de l'état technique du bâtiment de fabrication du l'acide chlorhydrique de synthèse, après plus de 35 ans de service dans le milieu très fortement agressif, a révélé l'existence des dégradations graves par corrosion des éléments structuraux en béton armé, qui affectent la résistance mécanique, la stabilité et la sécurité d'utilisation du bâtiment et qui constituent un risque potentiel d'effondrement du bâtiment et d'accidents. Les dégradations par corrosion des éléments de construction en béton armé consistent principalement en:

- aux poteaux du bâtiment (figure 3):

- la dégradation en profondeur du béton par corrosion acide, notamment à la base des poteaux, en dépassant le niveau des armatures dans plusieurs cas et en laissant découvertes les armatures en contact direct avec le milieu agressif;
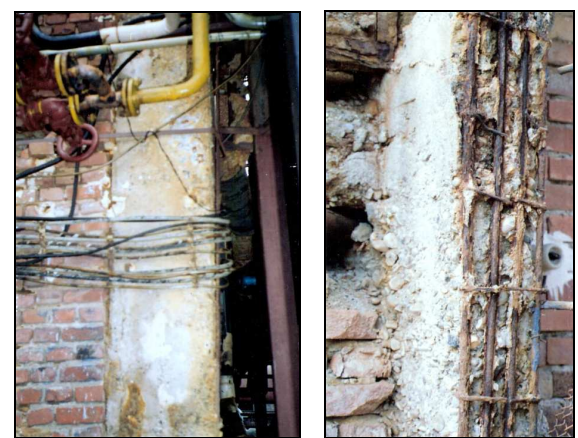

Fig. 3. Poteaux: dégradation en profondeur du béton par corrosion acide; corrosion avancée des armatures, avec réductions significatives de section/leur disparition sur certaines zones; armatures "flottantes".

- la corrosion avancée des armatures longitudinales et transversales (étriers), avec des réductions significatives de section, jusqu'à la disparition complète des armatures, sur certaines zones; aux certains poteaux, les armatures longitudinales sont devenues "flottantes" sur toute l'hauteur du poteau, suite à la disparition des étriers et la destruction par corrosion du béton d'enrobage;

- aux poutres (plancher de toiture, planchers technologiques) (figure 4):

- la fissuration du béton au long des armatures et l'éclatement du béton d'enrobage dus à la corrosion des armatures en acier;

- la corrosion avancée des armatures longitudinales et transversales (étriers), avec des réductions significatives de section, jusqu'à la disparition des étriers, sur certaines zones, par corrosion acide généralisée ("dissolution");
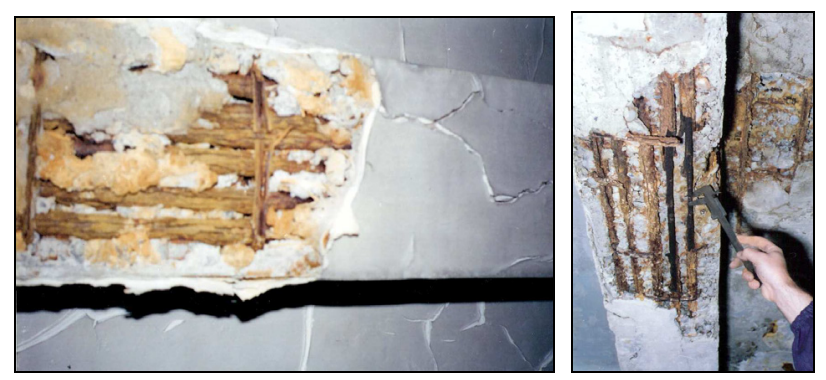

Fig. 4. Poutre de toiture/plateforme technologique: fissuration et éclatement du béton d'enrobage; corrosion avancée des armatures, avec des réductions significatives de section, jusqu'à la disparition des étriers, sur certaines zones.

- aux planchers technologiques:

- la fissuration et l'éclatement du béton d'enrobage dus à la corrosion avancée des armatures en acier, avec des réductions significatives de section, jusqu'à la disparition des étriers, sur certaines zones; corrosion acide du béton (figure 5);

- la dégradation complète du béton due à la corrosion acide dans la zone d'appui de la plaque sur le mur en maçonnerie (figure 6); corrosion avancée des armatures, avec des réductions importantes de leur section, jusqu'à la disparition des étriers, sur certaines zones;

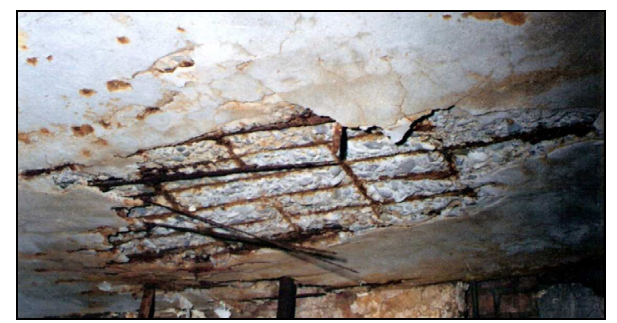

Fig. 5. Plancher technologique: fissuration et éclatement du béton d'enrobage; corrosion avancée des armatures, avec des réductions significatives de section, jusqu'à leur disparition.

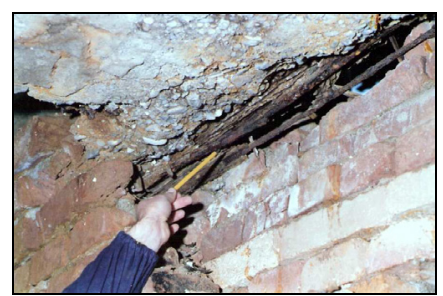

Fig. 6. Plancher technologique: dégradation complète du béton par corrosion dans la zone d'appui de la plaque sur le mur en maçonnerie; corrosion avancée des armatures, avec des réductions significatives de section.

- au plancher de toiture:

- dégradations prononcées par corrosion acide, tant de la plaque que des poutres en béton armé: la corrosion en profondeur du béton; corrosion avancée des armatures en acier qui a conduit à l'éclatement du béton d'enrobage.

Les dégradations graves existantes sont dus au service de longue durée des éléments de construction dans un milieu fortement agressif, constitué principalement de gaz, vapeurs et solutions acides $(\mathrm{HCl})$, suite au fonctionnement défectueux des installations technologiques (y compris la ventilation) et de la préoccupation insuffisante pour la réalisation des travaux de maintenance du bâtiment et des installations technologiques.

Les dégradations ont été favorisées et accentuées par un certain nombre de facteurs, tels que:

- l'utilisation à l'exécution du bâtiment des éléments en béton armé monolithe à défauts initiaux d'exécution (porosité élevée du béton - nids de cailloux, creux, cavernes) (dans le cas des poutres) et des éléments avec l'épaisseur insuffisante de la couche d'enrobage en béton par rapport au degré d'agressivité du milieu (dans le cas 
des tous éléments) ainsi que l'utilisation à l'exécution des certains éléments des bétons la classe de résistance et le degré d'imperméabilité inferieurs (dans le cas des poteaux);

- l'existence des trous technologiques dans la plaque des planchers, à travers lesquelles ont eu lieu des fuites de solutions agressives;

- l'absence des mesures efficientes de protection anticorrosive des éléments de construction par rapport à la nature et au degré d'agressivité du milieu de l'intérieur du bâtiment ainsi que l'inefficience des travaux de maintenance du bâtiment et des installations techniques.

Les processus de dégradation par corrosion des éléments structuraux en béton armé sont dus à l'action spécifique des agents agressifs à caractère fortement acide de l'intérieur du bâtiment, en affectant tant le béton (par corrosion acide) que les armatures en acier (par corrosion généralisée) et ayant comme résultat final la destruction complète des deux matériaux de construction.

Étant donné l'état de dégradation par corrosion très avancée de la construction, qui affectent la résistance mécanique, la stabilité et la sécurité d'utilisation du bâtiment, en existant le risque d'effondrement à toute surcharge accidentelle ou même en leur absence, on a proposé des mesures radicales d'intervention consistant en la démolition du bâtiment existant.

\section{Tests de laboratoire}

Les résultats des tests chimiques de laboratoire sur les échantillons de béton prélevés des éléments de construction dégradés par corrosion acide, ont montré les résultats suivants:

\subsection{Bâtiment de fabrication dans un milieu à base d'acide sulfurique}

- le pH de la suspension aqueuse des échantillons de béton dégradé a varié entre 1.0...2.5, les valeurs indiquant la désalcalinisation et destruction complète du béton par corrosion acide, ce qui confirme les résultats des tests effectués in situ;

- la concentration des ions sulfate $\left(\mathrm{SO}_{4}{ }^{2-}\right)$ solubles dans l'eau dans les échantillons de béton dégradé a variée entre $2.11 \ldots 19.2 \% \mathrm{SO}_{4}{ }^{2-}$, les valeurs auxquelles les ions $\mathrm{SO}_{4}{ }^{2-}$ ont un effet fortement corrosif, tant sur le béton que sur les armatures en acier.

\subsection{Bâtiment de fabrication dans un milieu à base d'acide chlorhydrique}

- le pH de la suspension aqueuse des échantillons de béton dégradé a varié entre 4.0...6.0, les valeurs indiquant la désalcalinisation totale du béton sous l'action de agents agressifs à caractère acide, ce qui confirme les résultats des tests effectués in situ;

- la concentration des ions $\mathrm{Cl}^{-}$solubles dans l'eau dans les échantillons de béton dégradé a variée entre 0.23 ... $2.98 \% \mathrm{Cl}^{-}$, les valeurs auxquelles les ions $\mathrm{Cl}^{-}$ont un effet fortement corrosif, spécialement sur les armatures en acier.

\section{Mécanismes de corrosion}

L'agressivité des milieux acides sur des éléments de construction en béton armé se manifeste complexe, en affectant tant le béton que l'armature en acier, par des mécanismes de corrosion différents.

\subsection{Corrosion du béton}

Les processus de corrosion du béton sous l'action des milieux acides se manifeste à un $\mathrm{pH}<6,5$ et se produit par réactions de double échange entre les constituants du milieu agressif et les composés hydratés du ciment, par la formation de certains produits de réaction très solubles (qui sont lessivés) ou peu solubles/insolubles (qui précipite sous forme de masse des gels, sans propriétés liantes).

Les acides forts tels que $\mathrm{HCl}$ dissolvent tous les composants de la pâte de ciment durci, par la formation des sels de calcium, aluminium et fer, selon les réactions chimiques suivantes:

$$
\begin{aligned}
& 2 \mathrm{HCl}+\mathrm{Ca}(\mathrm{OH})_{2} \rightarrow \mathrm{CaCl}_{2}+2 \mathrm{H}_{2} \mathrm{O} \\
& 4 \mathrm{mHCl}+\mathrm{m}\left(2 \mathrm{CaO} \cdot \mathrm{SiO}_{2} \cdot \mathrm{aq}\right) \rightarrow 2 \mathrm{mCaCl}_{2}+\mathrm{mSi}(\mathrm{OH})_{4} \\
& \mathrm{mSi}(\mathrm{OH})_{4} \rightarrow \mathrm{SiO}_{2} \cdot \mathrm{n} \mathrm{H}_{2} \mathrm{O}+2(2 \mathrm{~m}-\mathrm{n}) \mathrm{H}_{2} \mathrm{O} \\
& 8 \mathrm{HCl}+4 \mathrm{CaO} \cdot \mathrm{Al}_{2} \mathrm{O}_{3} \cdot 13 \mathrm{H}_{2} \mathrm{O} \rightarrow 4 \mathrm{CaCl}_{2}+2 \mathrm{Al}(\mathrm{OH})_{3}+ \\
& 14 \mathrm{H}_{2} \mathrm{O}
\end{aligned}
$$

Il en résulte que par la réaction directe des solutions acides avec les produits d'hydratation du ciment se forment des sels de calcium $\left(\mathrm{CaCl}_{2}\right.$ qui est très soluble) et, à la corrosion complète, des gels d'acide silicique, l'hydroxyde d'aluminium et l'hydroxyde de fer. L'hydroxyde d'aluminium et l'hydroxyde de fer réagissent avec des acides forts (par formations des sels) ou peuvent exister en tant que tels dans le cas d'acides faibles.

Un cas particulier est l'acide sulfurique $\mathrm{H}_{2} \mathrm{SO}_{4}$, dont l'action a un effet cumulatif sur la corrosion du béton: la corrosion acide, due aux ions d'hydrogène $\mathrm{H}^{+}$et la corrosion sulfatique, dues aux ions de sulfate $\left(\mathrm{SO}_{4}{ }^{2-}\right)$.

Dans le cas de $\mathrm{H}_{2} \mathrm{SO}_{4}$, se forme le sulfate de calcium qui précipite sous forme de gypse, par la réaction chimique directe de la forme:

$$
\mathrm{H}_{2} \mathrm{SO}_{4}+\mathrm{Ca}(\mathrm{OH})_{2} \rightarrow \mathrm{CaSO}_{4} \cdot 2 \mathrm{H}_{2} \mathrm{O}
$$

Le sulfate de calcium résultant peut cristalliser dans le béton (à des concentrations élevées de $\mathrm{H}_{2} \mathrm{SO}_{4}$ ) ou peut réagir avec les aluminates de calcium $\left(\mathrm{C}_{3} \mathrm{~A}\right)$ pour former des sulfo-aluminates de calcium hydratés (ettringite), ayant pour effet l'expansion du béton:

$$
3 \mathrm{CaSO}_{4} \cdot 2 \mathrm{H}_{2} \mathrm{O}+\mathrm{C}_{3} \mathrm{~A}+26 \mathrm{H}_{2} \mathrm{O} \rightarrow \mathrm{C}_{3} \mathrm{~A} .3 \mathrm{CaSO}_{4} .32 \mathrm{H}_{2} \mathrm{O} \text { (6) }
$$

La corrosion sulfatique se produit par un mécanisme d'expansion et a lieu par les réactions entre les sulfates du milieu agressif et l'hydroxyde de calcium libre et les aluminates de calcium hydratés de la pâte de ciment durci. Suite à ces réactions chimiques, se forment tant le sulfate de calcium (gypse) que plus particulièrement, le trisulfo-aluminate de calcium hydraté (ettringite), qui 
cristallise avec 32 molécules d'eau, ce qui produit une augmentation significative du volume (d'environ8 fois) et l'apparition de phénomènes forts d'expansion dans le masse du béton. Finalement, se produit la fissuration et, puis, la destruction rapide du béton. À des concentrations élevées de sulfates, précipite aussi le gypse, qui présente également une augmentation de volume.

De même agit l'acide sulfureux $\mathrm{H}_{2} \mathrm{SO}_{3}$, l'hydrogène sulfuré $\mathrm{H}_{2} \mathrm{~S}$, le dioxyde $\mathrm{SO}_{2}$ et le trioxyde de soufre $\mathrm{SO}_{3}$, dans une atmosphère humide, parce que toutes ces substances peuvent être oxydés, pour former $\mathrm{H}_{2} \mathrm{SO}_{4}$.

\subsection{Corrosion de l'armature en acier}

Le processus de corrosion de l'armature en acier est de nature électrochimique, étant le résultat du déroulement simultané de deux types de réactions aux électrodes, anodiques et cathodiques. Simplifié, le mécanisme de corrosion des armatures en acier est décrit par les deux réactions [7]:

- la réaction anodique, de dissolution (oxydation) du fer:

$\mathrm{Fe} \rightarrow \mathrm{Fe}^{2+}+2 \mathrm{e}^{-}$

- les réactions cathodiques, de réduction des ions d'hydrogène ou d'oxygène (en milieu acide):

$$
\begin{aligned}
& 2 \mathrm{H}^{+}+2 \mathrm{e}^{-} \rightarrow \mathrm{H}_{2} \\
& \mathrm{O}_{2}+4 \mathrm{H}^{+}+4 \mathrm{e}^{-} \rightarrow 2 \mathrm{H}_{2} \mathrm{O}
\end{aligned}
$$

Dans la plupart des cas, le processus de corrosion de l'acier dans des milieux acides se déroule avec une dépolarisation d'hydrogène.

La corrosion de l'armature dépend tant des propriétés du béton et de l'acier, que la nature et le caractère du milieu dans lequel est situé l'élément de construction.

Dans des conditions normales, l'armature d'acier est protégée dans le béton sain par le phénomène de passivation grâce au $\mathrm{pH}$ élevé $(\mathrm{pH}=12 \ldots 13)$ de la solution interstitielle contenue dans les pores du béton. À ces valeurs de $\mathrm{pH}$, la formation d'une couche protectrice microscopique sur la surface de l'acier ne permet pas la corrosion (dissolution du fer), même en présence d'humidité et d'oxygène.

La couche de passivation de l'acier dans le béton peut être détruite, respectivement la dépassivation et la corrosion de l'acier deviennent possibles dans diverses conditions:

- la réduction de l'alcalinité du béton par l'action d'agents agressifs à caractère acide (ex. carbonatation par $\mathrm{CO}_{2}$ ) jusqu' aux valeurs du $\mathrm{pH}$ du béton inférieur à environ 9; - la présence des anions dépassivants $\left(\mathrm{Cl}^{-}, \mathrm{SO}_{4}{ }^{2-}, \mathrm{NO}_{3}{ }^{-}\right.$ etc.) à concentrations critiques au niveau de l'armature; - la destruction par corrosion de la couche d'enrobage en béton;

- la présence des fissures dans le béton etc.

Dans le cas des gaz et vapeurs acides $\left(\mathrm{Cl}_{2}, \mathrm{HCl}\right.$, etc. $)$ qui diffusent facilement à travers du béton, la corrosion de l'armature peut se produire avant la carbonatation / désalcalinisation totale de la couche d'enrobage en béton. L'aspect extérieur de la corrosion du béton armé provoquée par $\mathrm{Cl}_{2}$ et $\mathrm{HCl}$ gazeux (en présence de l'humidité atmosphérique), ainsi que par les composés du chlore en général, est la fissuration du béton au long des armatures à cause de la pression des produits de corrosion de l'acier (rouille) sur la couche d'enrobage en béton.

Dans le cas des solutions acides, la corrosion de l'armature d'acier se produit après la désalcalinisation complète de la couche d'enrobage en béton.

En général, dans les milieux agressifs acides se produit une corrosion généralisée ou localisée de l'acier, avec des réductions significatives de section des armatures (parfois même leur "dissolution" complete). Une dernière forme de corrosion est induite par l'action simultanée de la corrosion et d'une sollicitation mécanique. Il s'agit de corrosion fissurante sous tension induite par l'hydrogène (fragilisation par l'hydrogène) qui est caractéristique pour les aciers à haute résistance pour la précontrainte du beton dans certains milieux agressifs spécifiques, tels que les milieux contenant ions $\mathrm{SH}^{-}, \mathrm{SCN}, \mathrm{H}^{+}$etc.). Cette forme insidieuse de corrosion, caractérisée par la fissuration et la fracture brusque de l'armature en acier prétendue, sans striction et sans perte visible de métal, est très dangereuse parce que peut provoquer l'avarie des éléments/constructions en béton précontraint sans l'apparition au préalable des signes extérieurs avertissant le danger [8].

Dans le cas de l'action des anions corrosifs, tels que des ions sulfate $\left(\mathrm{SO}_{4}{ }^{2-}\right)$, la couche protectrice existante sur la surface de l'armature dans le béton est détruite localement et provoque la corrosion localisée de l'acier. Étant donné que la zone anodique est beaucoup plus réduite par rapport à la zone cathodique se produit une diminution significative de la section de l'armature dans cette zone. Par rapport aux ions $\mathrm{Cl}^{-}$, les ions $\mathrm{SO}_{4}{ }^{2-}$ ont une action corrosive inférieure sur l'armature en acier; le sulfate de fer formé initialement se transforme en rouille en présence de l'oxygène et de l'eau. À des concentrations élevées des ions $\mathrm{SO}_{4}{ }^{2-}$ se produit la corrosion généralisée de l'armature en acier.

La diminution de section de l'armature en acier et le gonflement simultané de la rouille entraînent une diminution plus ou moins notable de l'adhérence entre l'armature en acier et le béton.

\section{Conclusions}

Les résultats des recherches sur le comportement en service de longue durée des éléments en béton armé qui constituent la structure de résistance des certaines constructions soumises à l'action corrosive des milieux agressifs acides ont révélé l'existence des dégradations graves par corrosion des éléments / constructions, qui ont conduit à la réduction significative ou même à la perte de la capacité portante des certains éléments structuraux et qui constituent des risques potentiels d'effondrement des constructions et d'accidents.

Les dégradations par corrosion ont été causées par les conditions sévères de service des éléments / constructions dans des milieux acides fortement agressifs, étant favorisées et accentuées par l'absence des mesures efficientes de protection anticorrosive des éléments de construction par rapport à la nature et au degré 
d'agressivité des milieux de l'intérieur des constructions ainsi que de l'inefficience des travaux de maintenance des constructions et des installations technologiques.

Afin d'intervenir avec des mesures efficientes de prévention de la dégradation des éléments de construction soumis à l'action des milieux agressifs acides est nécessaire de bien connaître les mécanismes des processus de corrosion des matériaux de construction (béton, armatures en acier).

Étant donné l'état de dégradation très avancée par corrosion des constructions, qui affectent leur résistance mécanique, stabilité et sécurité d'utilisation, on a proposé des mesures radicales d'intervention consistant en démolition des constructions dégradées existantes et la réalisation de nouvelles constructions, conçues et construites conformément aux exigences fondamentales applicables aux ouvrages de construction imposées par les processus technologiques de fabrication, qui doivent être modernisés également.

\section{References}

1. I. Pepenar, Conferinta Nationala Comportarea in situ a constructiilor, Romania, 57 (1994)

2. I. Pepenar, Constructii, 3, 40 (1977)

3. I. Pepenar, Constructii, 11, 33 (1979)

4. I. Pepenar, Durability of Building Materials and Components 8, Ottawa, Canada, 231 (1999)

5. NE 012/1, Normativ pentru producerea betonului si executarea lucrarilor din beton, beton armat si beton precomprimat, Partea 1: Producerea betonului, Anexa I, Romania (2007)

6. Règlement (UE) $\mathrm{N}^{\mathrm{o}} 305 \mathrm{du}$ Parlement Européenne et du Conseil, JO L 88 du 4.4.2011 (2011)

7. I. Pepenar, G. Grimaldi, A. Raharinaivo, Études et recherches des LPC, OA 14, 17 (1994)

8. I. Pepenar, Durability of Construction Materials, Paris, France, 1388 (1987) 